\title{
EL TRABAJO COLABORATIVO COMO HERRAMIENTA PARA EL DESARROLLO PERSONAL. UNA EXPERIENCIA EN UNA ASIGNATURA DE MARKETING
}

\section{COLLABORATIVE WORK AS A TOOL FOR PERSONAL DEVELOPMENT. AN EXPERIENCE IN A MARKETING SUBJECT}

\author{
Elena González-Gascón1: Universidad Miguel Hernández de Elche. España. \\ elena.gonzalez@umh.es
}

\section{RESUMEN}

La enseñanza universitaria está evolucionando desde un modelo tradicional individualista, basado en la transmisión y reproducción de conocimientos a un nuevo modelo, en el que la implicación del estudiante en las tareas a realizar es total. El Espacio Europeo de Estudios Superiores establece, entre otras, la competencia transversal de trabajo colaborativo. El trabajo colaborativo es un método de enseñanza basado en el trabajo en grupo, que persigue una mejora del rendimiento y de la interacción de los estudiantes. Siendo una de sus principales características la construcción del consenso, a través de la colaboración de los miembros del grupo, donde la autoridad es compartida y todos aceptan la responsabilidad de las acciones del equipo. El trabajo colaborativo se centra tanto en las ventajas cognitivas derivadas de los intercambios de conocimientos, opiniones y perspectivas que se producen al trabajar en equipo como en conseguir una mejora en las relaciones sociales. En este trabajo se muestra la aplicación del trabajo colaborativo como metodología docente en una asignatura de marketing. Los estudiantes consiguen tanto adquirir los resultados de aprendizaje y desarrollar las competencias establecidas como mejorar las relaciones sociales del grupo e incrementar su desarrollo personal.

PALABRAS CLAVE: docencia, trabajo colaborativo, desarrollo personal, marketing.

\section{ABSTRACT}

University education is evolving from a traditional individualistic model, based on the transmission and reproduction of knowledge to a new model, in which the student involvement in the tasks is total. The European Higher Education Area provides, inter alia, the cross competition for collaborative work. Collaborative work is a teaching method based on group work, pursuing an improvement in performance, and student interaction. As one of its main characteristics consensus building, through the collaboration of members of the group, where authority is shared and everyone accepts responsibility for the team's actions. Collaborative work focuses both, on the cognitive benefits of the exchange of information, views and perspectives that occur when working in teams; and achieve an improvement in social relations. This paper

${ }^{1}$ Elena González-Gascón: Departamento de Estudios Económicos y Financieros, profesor colaborador (Área de Comercialización e Investigación de Mercados). 
shows the application of collaborative work as a teaching methodology in a marketing subject. Students get acquire both, learning outcomes and develop the established competencies and improving social relations of the group, and increase their personal development.

KEY WORDS: teaching, collaborative work, personal development, marketing.

\section{Cómo citar el artículo:}

González-Gascón, E. (2017). El trabajo colaborativo como herramienta para el desarrollo personal. Una experiencia en una asignatura de marketing. Revista de Ciencias de la Comunicación e Información, 22(2), 75-88.

doi: http://doi.org/10.35742/rcci.2017.22(2).75-88

\section{INTRODUCCIÓN}

La enseñanza universitaria está evolucionando desde un modelo tradicional individualista, basado en la transmisión y reproducción de conocimientos y contenidos, a nuevos escenarios en los que la implicación de los estudiantes en las tareas a realizar es total. El objetivo es convertirlos en sujetos activos de la construcción y gestión de su propio aprendizaje (Heredia Avalos et. al., 2013) consiguiendo así un mayor desarrollo personal.

La reforma educativa introducida por el proceso de convergencia europeo propone entender el aprendizaje desplazando la responsabilidad y control del mismo a los propios estudiantes. El rol del profesor cambia, dejando de ser un transmisor de conocimientos para convertirse en quien facilita el uso de recursos y herramientas, ayuda a los estudiantes a reflexionar y plantearse nuevas preguntas, y a relacionar las informaciones, guiando el proceso de construcción del conocimiento, fomentando la interacción y creatividad individual y grupal, la innovación y la investigación (Zubimendi-Herranz et al., 2010).

El Espacio Europeo de Estudios Superiores establece, entre otras, la competencia transversal de trabajo colaborativo, cuyo objetivo es promover un aprendizaje autónomo, comprometido y adaptado las nuevas necesidades de las empresas y de la sociedad del siglo XXI (Ibarra Saiz y Rodríguez Gómez, 2007).

El trabajo colaborativo es un método de enseñanza basado en el trabajo en grupo, que persigue una mejora del rendimiento y de la interacción de los estudiantes (Cabero, 2003).

Una de las principales características del trabajo colaborativo se basa en la construcción del consenso, a través de la colaboración de los miembros del grupo, donde la autoridad es compartida y todos aceptan la responsabilidad de las acciones del equipo (Panitz, 1995). El trabajo colaborativo se centra en las ventajas cognitivas derivadas de los intercambios de conocimientos, opiniones y perspectivas que se producen al trabajar en equipo (Slavin, 1995) además persigue una mejora en las relaciones sociales, no solo entre docente y discente, sino también entre los discentes (Serrano y González-Herrero, 1996). 
Cuando el docente se plantea introducir en la planificación docente de una asignatura, el trabajo colaborativo, debe saber que, normalmente, conlleva una mayor carga de trabajo para el mismo (con una importante dedicación en tiempo y esfuerzo para el seguimiento y evaluación de las diferentes etapas del trabajo realizado), pero también supone un esfuerzo muy importante para los estudiantes (que han de participar activa y responsablemente en los trabajos de aprendizaje planteados, cumpliendo los plazos y objetivos fijados) (Echazarreta et al., 2009).

Tanto el docente como el discente deben conocer algunas cuestiones básicas sobre el trabajo colaborativo para que la asignatura se desarrolle según lo planeado y se alcancen tanto los objetivos de aprendizaje como las competencias planteadas. En la Tabla 1 se pueden observar las principales características del trabajo colaborativo y las exigencias que suponen a nivel individual y grupal, que deben conocer todos los implicados para el buen desarrollo de las actividades.

Tabla 1. Cuestiones básicas sobre el trabajo colaborativo

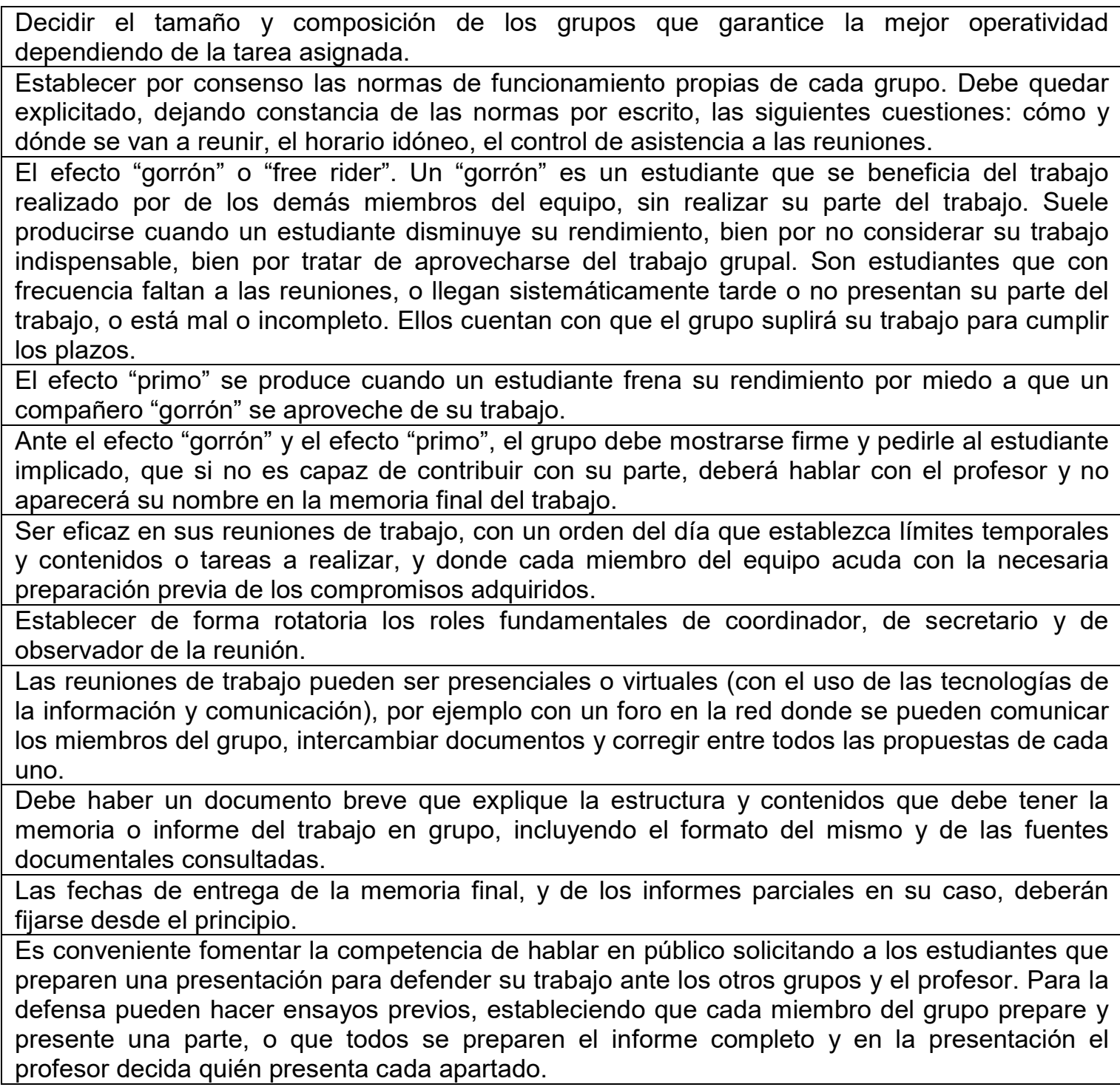

Fuente: Elaboración propia basado en Heredia Avalos et. al., (2013). 


\section{OBJETIVOS}

El objetivo de este trabajo es doble. Por un lado mostrar una experiencia docente, en la que a través del trabajo colaborativo los estudiantes adquieren los conocimientos y las competencias especificadas en la guía docente de la asignatura "Marketing en Biotecnología". Por otro, exponer como el trabajo colaborativo es una buena herramienta docente que contribuye no solo a la adquisición de competencias sino al desarrollo personal de los estudiantes.

En concreto, uno de los retos de esta experiencia ha sido comprobar si los estudiantes adquieren las competencias y alcanzan los resultados de aprendizaje, a través del trabajo colaborativo, siendo conscientes en todo momento de que no es necesario realizar la prueba escrita de conocimientos, es decir el típico examen, si superan las prácticas de la asignatura, realizadas obligatoriamente con trabajo colaborativo.

\section{METODOLOGÍA}

\subsection{Descripción de la asignatura Marketing en Biotecnología}

En este apartado se expone la metodología utilizada para impartir la asignatura de Marketing en Biotecnología (MktBio), centrada en su mayor parte en el trabajo colaborativo. MktBio es una asignatura optativa de cuarto curso del Grado en Biotecnología de la Universidad Miguel Hernández de Elche. Se imparte en el segundo cuatrimestre, tiene seis créditos ECTS, tres de teoría y tres de prácticas.

De las 12 competencias generales del grado, a continuación se enumeran las que se trabajan en la asignatura MktBio:

- Adquisición de conocimiento científico básico.

- Capacidad de identificación, formulación y resolución de problemas básicos.

- Capacidad para la mejora continua, la experimentación y la innovación.

- Capacidad para la evaluación, optimización y confrontación de criterios para la toma de decisiones.

- Capacidad para la redacción, representación, análisis e interpretación de documentación científica y de datos relevantes en el ámbito de la rama de Ciencias.

- Capacidad de transmisión de conocimientos adquiridos en ambientes de expertos y no expertos.

- Capacidad de trabajo en equipos multidisciplinares y multiculturales.

- Compromiso social, ético y medioambiental para el desarrollo de soluciones científicas, compatibles y sostenibles con la realidad del entorno humano y natural.

- Capacidad de adaptación y actualización de nuevos conocimientos y avances científicos, adoptando una actitud ética, de innovación y creatividad en el ejercicio de la profesión.

Como competencia específica figura "saber difundir las ventajas y limitaciones de los productos biotecnológicos", siendo los objetivos o resultados de aprendizaje que permiten alcanzar la competencia específica los siguientes: 
- Valorar el alcance y la importancia del marketing para realizar intercambios en los mercados.

- Analizar las oportunidades de marketing que ofrece el mercado.

- Comprender el comportamiento de los consumidores y aplicar este conocimiento para lograr intercambios rentables.

- Identificar, analizar y tomar decisiones acerca del conjunto de actividades de las políticas de producto, precio, distribución y comunicación comercial a disposición de la empresa.

- Analizar las situaciones reales de los mercados, para aplicar los conceptos, principios, modelos y herramientas de la dirección de marketing, resolver problemas comerciales y ayudar al logro de los objetivos de la organización.

- Ser capaz de elaborar un plan de marketing.

Los contenidos de la asignatura se organizan en seis unidades didácticas (a) el comportamiento del consumidor; b) decisiones sobre productos biotecnológicos; c) decisiones sobre precio en productos biotecnológicos; d) decisiones sobre distribución de productos biotecnológicos; e) decisiones sobre comunicación de productos biotecnológicos, y f) el programa comercial) con el objetivo de facilitar el trabajo de los contenidos que permiten alcanzar los resultados de aprendizaje planteados.

En cuanto a la metodología utilizada se ha utilizado la siguiente:

- Aprendizaje basado en problemas: Desarrollar aprendizajes activos a través de la resolución de problemas que fomenten en el estudiante el pensamiento y/o experimentación, así como la toma de decisiones.

- Aprendizaje colaborativo: Desarrollar aprendizajes activos mediante estrategias de trabajo colaborativo entre estudiantes y fomentando la responsabilidad compartida para alcanzar metas grupales.

- Aprendizaje orientado a proyectos: Realización de un proyecto para la resolución de un problema, aplicando aprendizajes adquiridos y fomentando habilidades relacionadas con la planificación, diseño, realización de actividades y obtención de conclusiones.

- Expositivo/Lección magistral: Transmitir conocimientos y activar procesos cognitivos en el estudiante, implicando su participación.

- Resolución de ejercicios y problemas: Ejercitar, ensayar y poner en práctica los conocimientos previos mediante la repetición de rutinas.

La evaluación de la asignatura está diseñada para promover el trabajo colaborativo, pero también da la oportunidad, a aquellos estudiantes que de manera voluntaria o de manera obligatoria queden fuera del mismo, a aprobar la misma. Para ello se presenta a los estudiantes un sistema dual, o bien siguen un proceso de evaluación continua o bien se presentan a una evaluación final. La evaluación continua exige la realización, entrega y defensa de todas las prácticas obligatorias realizadas a lo largo del curso (un total de seis). La nota de la asignatura está formada por la media de las notas obtenidas por el estudiante en las prácticas realizadas. La evaluación final, para el estudiante que no haya entregado las prácticas, se realiza con una prueba escrita, que puede ser teórica o/y práctica, que consta de 5 preguntas sobre los contenidos de la asignatura. 
En cuanto a la planificación temporal de la asignatura, las 15 semanas lectivas se han repartido de la siguiente manera. La primera semana se ha dedicado a explicar la metodología docente de la misma y a informar a los estudiantes sobre las características del trabajo colaborativo, dejando que ellos de manera libre formen los equipos de trabajo, con la única limitación del número de componentes del mismo, que debe oscilar entre tres y cuatro componentes.

Para cada una de las seis unidades didácticas se han utilizado dos semanas, de las cuales la primera se ha dedicado a explicar la teoría de la asignatura, y la siguiente a realizar las prácticas de la unidad correspondiente.

Las dos semanas restantes, que se han situado al final de la unidad didáctica 3 y de la 6 , se han empleado en tomar el pulso a la asignatura, comprobando la adecuación de la metodología docente empleada y el ambiente general del grupo. También se han realizado evaluaciones grupales, entre pares y con el docente.

\subsection{Descripción de los estudiantes}

De los 21 estudiantes matriculados en la asignatura, el $62 \%$ son mujeres y el $38 \%$ son hombres. Todos ellos con edades que oscilan entre 21 y 23 años.

El estilo de aprendizaje que se utiliza en el Grado de Biotecnología es básicamente individual y excepcionalmente competitivo. Desde el primer día de clase, en la apertura del curso, se les dice a todos los estudiantes que el sistema de evaluación es competitivo, por tanto que sus compañeros de clase son rivales y que están compitiendo entre ellos. Se les anima a mantener actitudes como por ejemplo no prestarse apuntes, o no formar grupos de trabajo más allá de los necesarios para algunas prácticas grupales (que siempre son dirigidas y en las que, normalmente, los alumnos no escogen con que compañeros las realizan).

Aunque algunos estudiantes no adoptan de inmediato este estilo de trabajo, todos toman clara conciencia de que son rivales unos de otros al llegar al segundo curso del grado, donde se empiezan a ofertar prácticas en laboratorios, empresas y/o grupos de investigación y los estudiantes son elegidos por expediente académico.

De esta manera los estudiantes mantienen actitudes claramente individualistas y competitivas.

El Grado de Biotecnología en la Universidad Miguel Hernández de Elche tuvo el último curso una nota de corte de 11,251, es decir alta. Los estudiantes que acceden a la titulación tienen una trayectoria personal buena, están acostumbrados trabajar y obtener buenos rendimientos. Tras los 3 cursos en el grado, los estudiantes han adquirido rutinas de trabajo y se puede decir que son "buenos estudiantes".

\subsection{Descripción de las rutinas de trabajo de los grupos en las sesiones prácticas}

Como se ha comentado con anterioridad, en los criterios de evaluación, los estudiantes pueden elegir entre la evaluación continua y la final. Todos han elegido 
la primera opción y por tanto han formado los equipos de trabajo. Se forman 7 grupos de trabajo, tres de cuatro componentes y otros tres de tres.

Se advierte a los estudiantes que los grupos de trabajo son permanentes, de tal manera que los cambios no están permitidos y en caso de producirse la baja de alguno de los miembros del equipo, por el motivo que sea, el resto de los componentes del grupo deben continuar adelante, asumiendo una mayor carga de trabajo.

También se les informa que para cada práctica deben entregar un informe por escrito, presentarlo oralmente y defenderlo ante sus compañeros de clase y el docente. La presentación y defensa del informe se realiza en su totalidad por un solo componente del grupo, que es elegido en el mismo momento de la defensa de manera aleatoria por el docente, de esta forma, todos deben preparar tanto la presentación de cada práctica como su defensa. La nota obtenida, tanto en el informe escrito como en la presentación y defensa es solidaría, es decir, se comparte con el grupo.

En la primera sesión de trabajo cada grupo elige de manera libre un producto biotecnológico sobre el que trabajar a lo largo del curso. En cada una de las sesiones prácticas, los estudiantes deben responder a una serie de preguntas sobre la unidad didáctica correspondiente, aplicando los conocimientos adquiridos en las sesiones teóricas a casos concretos sobre productos biotecnológicos. En unos casos los elegidos por ellos mismos, en otros casos los elegidos por los compañeros de otros grupos. Al final del cuatrimestre todos los equipos han trabajado sobre todos los productos.

Una vez elaborado el informe, se procede a preparar su presentación y defensa. Todos los grupos de trabajo realizan preguntas a los compañeros que exponen. Una vez terminada la fase de defensa, los grupos de trabajo vuelven a reunirse con el objetivo de preparar una evaluación argumentada, utilizando unas rúbricas para ello, del trabajo de uno de los otros grupos. Esa evaluación, que no es pública, se entrega al docente.

El docente, para cada práctica, evalúa el informe escrito entregado, la presentación y la defensa realizada y la evaluación efectuada por el grupo. Una vez terminado todo el proceso, se pone en común con los estudiantes.

En la primera clase, tras la presentación de la asignatura se pasa un breve cuestionario anónimo a los estudiantes donde se les pregunta sobre la motivación que han tenido para elegir la optativa y sobre las expectativas que tienen de la misma, es decir sobre lo que esperan de la asignatura MktBio y lo que quiere de ella.

En la última sesión del curso, sin previo aviso, se les pasa a los estudiantes dos cuestionarios anónimos. En uno se les solicita que indiquen de manera priorizada, las tres cosas que más les han gustado de la asignatura y las tres que menos les han gustado o que cambiarían para el curso siguiente.

Una vez realizado el primer cuestionario, se les pasa el segundo, que es un examen tipo test, con 20 preguntas y tres opciones de respuesta cada una. Se les 
informa que el examen es anónimo, no habiendo espacio reservado para poner nombre, DNI o número de expediente. Igualmente se les informa que el objetivo de realizar la prueba de conocimientos es controlar si, el grupo en su conjunto ha alcanzado el nivel adecuado en los resultados de aprendizaje, pero que sea cual sea el resultado no va a afectar a la nota obtenida en la asignatura. Para finalizar se solicita su colaboración, pidiéndoles que realicen el examen como si fuera real, es decir, como si la nota obtenida en el mismo fuera a formar parte de la nota final.

\section{RESULTADOS}

En cuanto a las expectativas que tienen sobre la asignatura, es decir qué quieren y qué esperan de ella, el $82 \%$ tiene una motivación pragmática (un $71 \%$ quieren aprender y un $11 \%$ quieren "nota"), mientras que el $18 \%$ tiene una motivación hedónica.

En el Gráfico 1 se puede observar que del $71 \%$ de los estudiantes que declaran: "que quieren aprender", el argumento más utilizado: "la preparación para el futuro laboral".

Gráfico 1. Motivación pragmática. Aprender.

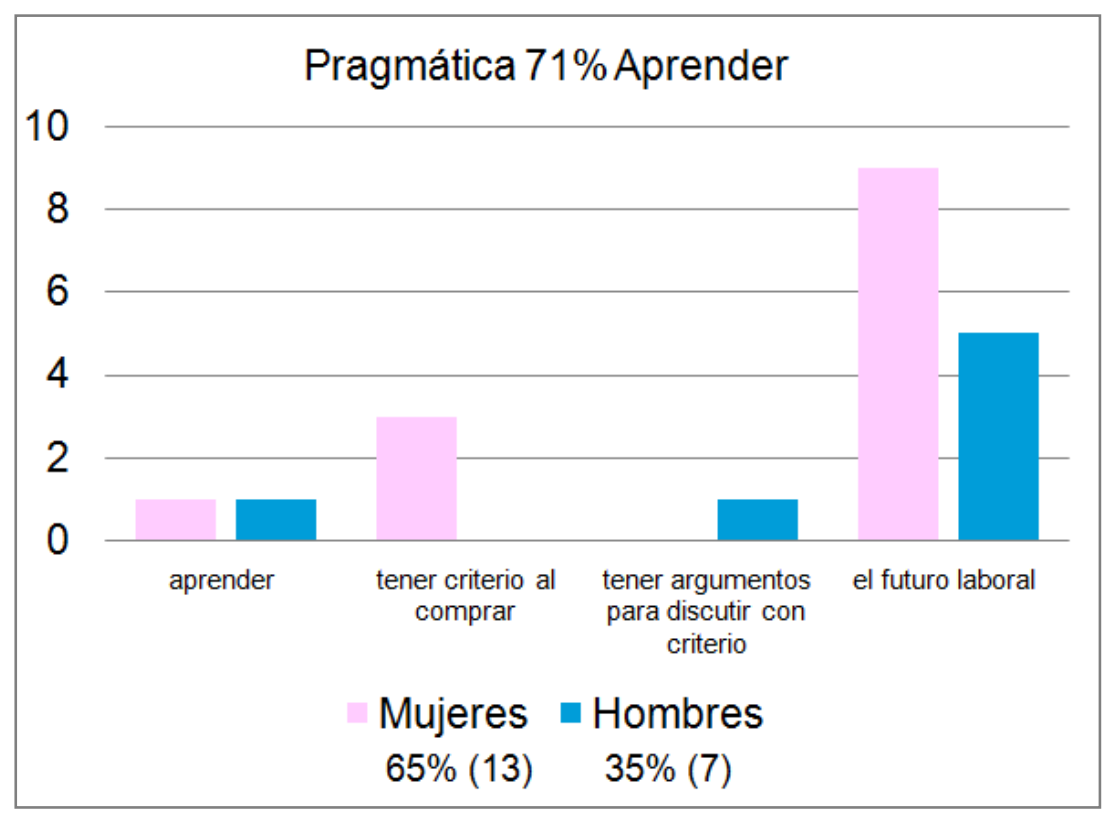

Fuente: Elaboración propia.

Como se observa en el Gráfico 2 el 18\% de los estudiantes, en este caso mujeres, manifiestan una motivación hedónica, declarando que: "quieren que la asignatura sea divertida, amena, participativa". 
Gráfico 2. Motivación hedónica.

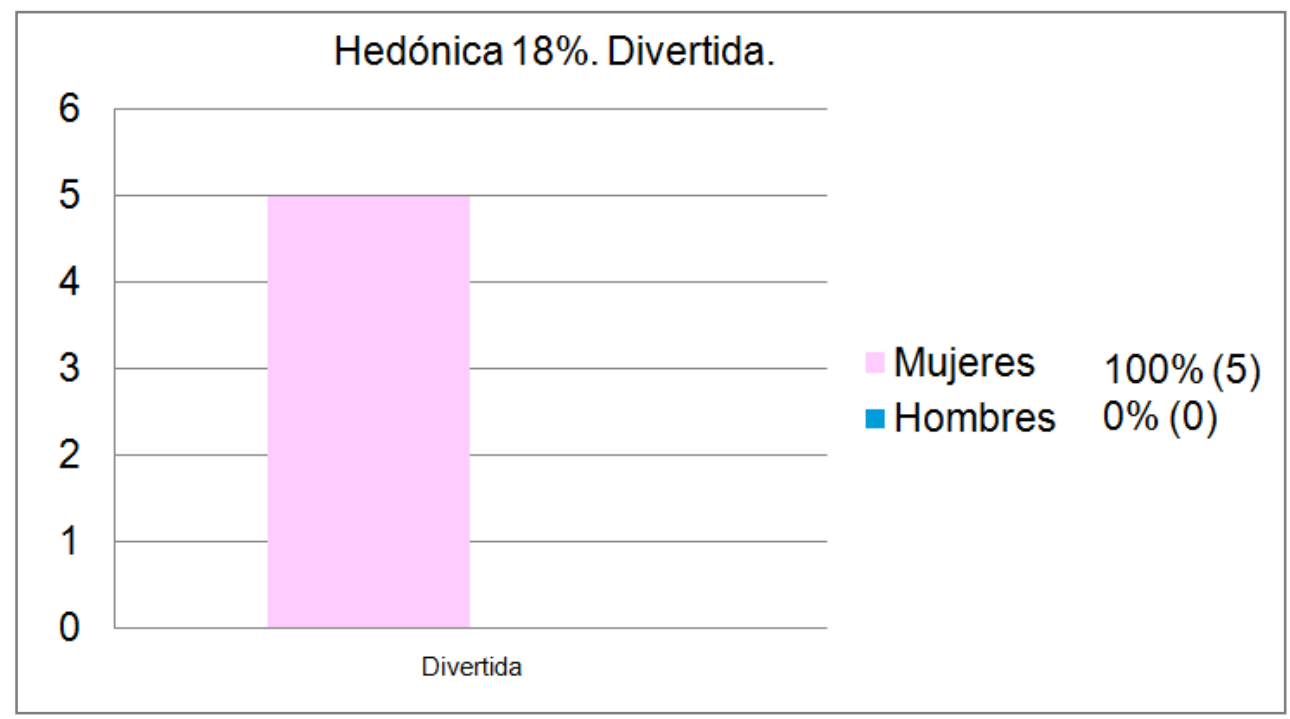

Fuente: Elaboración propia.

En el Gráfico 3 se puede observar que el 11\% de los estudiantes, manifiestan una motivación pragmática, con dos intereses diferentes, unos quieren "mejorar el expediente", sacando buenas notas y otros buscan "una asignatura que sea fácil de aprobar".

Gráfico 3. Motivación pragmática. Nota.

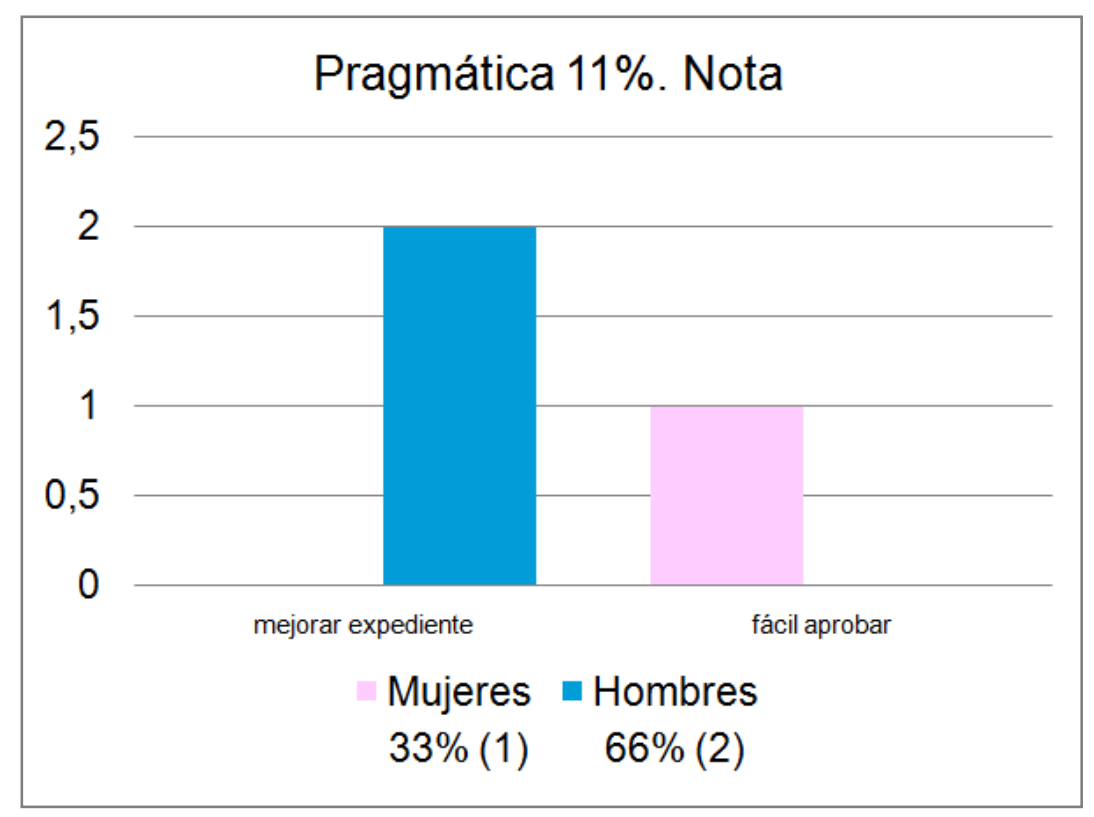

Fuente: Elaboración propia.

En la Tabla 2 se muestran tanto las notas finales como las notas de la prueba de conocimientos escrita. En el primer caso se trata de las notas finales obtenidas por 
los estudiantes al finalizar la asignatura, utilizando como metodología docente el trabajo colaborativo. Las notas están formadas por la media de las prácticas entregadas, teniendo en cuenta tanto el informe escrito, como su presentación y defensa y la evaluación realizada a los otros grupos de la clase. Para el segundo caso, se muestran las notas obtenidas por los estudiantes en la prueba de conocimientos escrita, realizada sin previo aviso el último día de clase. El examen era de tipo test, con 20 preguntas de tres opciones cada una, donde dos respuestas erróneas anulan una correcta. En ambos casos las notas se muestran ordenadas de mayor a menor, para que resulte más fácil la comparación de las mismas.

Tabla 2. Notas obtenidas por los estudiantes.

\begin{tabular}{|r|r|r|r|r|r|}
\hline \multicolumn{1}{|l|}{ Estudiante } & \multicolumn{1}{l|}{ Nota Final } & \multicolumn{1}{l|}{ Nota Test } & \multicolumn{1}{l|}{ Estudiante } & \multicolumn{1}{l|}{ Nota Final } & \multicolumn{1}{l|}{ Nota Test } \\
\hline 1 & 9,5 & 10 & 12 & 9,0 & 7,5 \\
\hline 2 & 9,5 & 9,5 & 13 & 8,9 & 7,5 \\
\hline 3 & 9,5 & 9 & 14 & 8,8 & 7,5 \\
\hline 4 & 9,4 & 9 & 15 & 8,8 & 7,5 \\
\hline 5 & 9,4 & 9 & 16 & 8,8 & 7,5 \\
\hline 6 & 9,4 & 9 & 17 & 8,7 & 7 \\
\hline 7 & 9,3 & 8,5 & 18 & 8,6 & 7 \\
\hline 8 & 9,3 & 8,5 & 19 & 8,6 & 7 \\
\hline 9 & 9,3 & 8,5 & 20 & 8,6 & 6 \\
\hline 10 & 9,2 & 8 & 21 & 8,4 & \\
\hline 11 & 9,2 & 7,5 & & & \\
\hline
\end{tabular}

En el Gráfico 4 se puede observar la diferencia entra la nota máxima, la mínima y la nota media del grupo, con los dos sistemas de evaluación. En el caso de la metodología de trabajo colaborativo, los alumnos saben en todo momento que están siendo evaluados, así como las fechas y los criterios de evaluación, siendo ésta además continua. La nota media del grupo es de 9,1 siendo la mínima de 8,4 y la máxima de 9,5. En el caso de la prueba de conocimientos escrita, fue realizada sin previo aviso el último día de clase y de manera individual. La nota media del grupo es de 8 siendo la mínima de 6 y la máxima de 10.

Gráfico 4. Comparativa de las notas obtenidas.

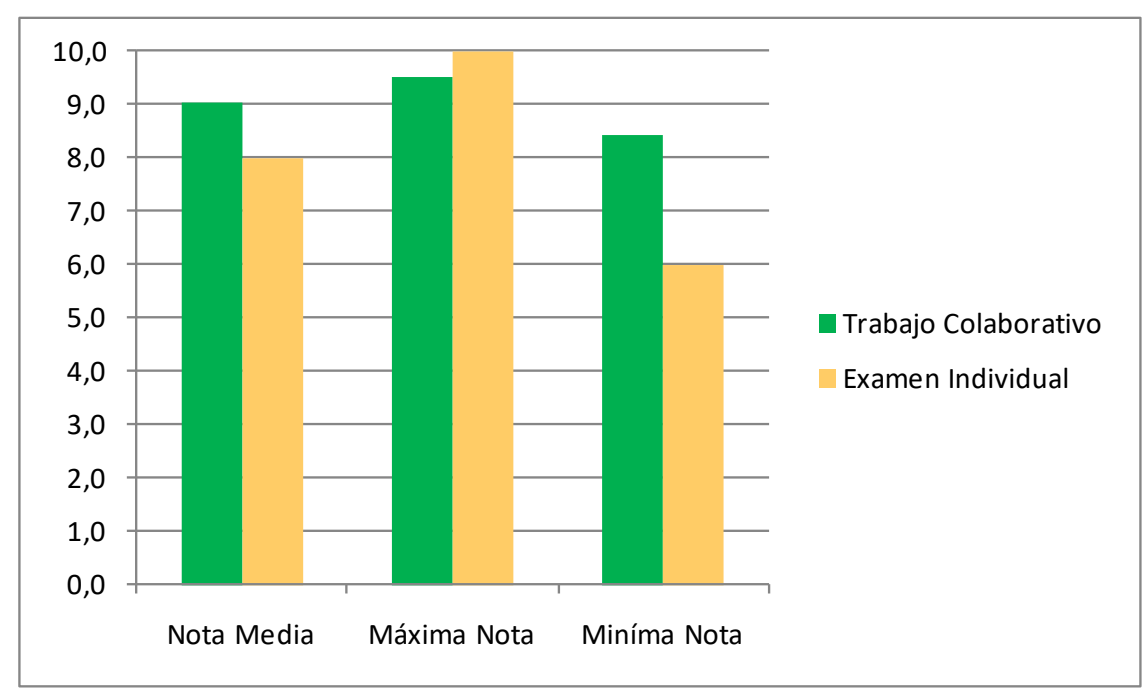


A continuación se reflejan algunas de las opiniones que los estudiantes escriben como respuesta al cuestionario sobre los aspectos que más les han gustado de la asignatura y aquellos que se pueden mejorar. La mayoría indica los mismos conceptos y sentimientos, explicados de diferentes maneras. Las siguientes opiniones son favorables a la experiencia docente vivida, basada en la metodología de trabajo colaborativo.

Estudiante 1: "El formato de las clases ha sido lo que más me ha gustado. Son clase dinámicas, participativas y al igual que en las prácticas, siempre hay alguna actividad extra que reduce la monotonía de una clase normal. También destacar el aprendizaje en exposiciones orales que esto supone".

Estudiante 2: "Las clases son entretenidas, participativas, dinámicas. Aprender a trabajar en equipo en las prácticas me ha gustado mucho".

Estudiante 3: "Las prácticas y su contenido. El poder combinar la visión biotecnológica de un producto con todo el marketing que hay detrás. El tener que trabajar "a tope" en clase. Con esto hemos aprendido a pensar, planificar y llevar a cabo tareas bajo presión y estrés".

Estudiante 4: "Clases dinámicas, en clase hay mucha participación y trabajo en equipo".

Estudiante 5: "El dinamismo de las clases, se hacian entretenidas, aun siendo clases de 2 horas o 2 horas y media. La dinámica y ambiente de trabajo, los conceptos quedaban más claros al hacer las prácticas y poder aplicar los conceptos teóricos de cada tema. Buen ambiente entre compañeros $y$ profesora".

Estudiante 6: "Lo mejor ha sido el modo en que hemos trabajo en clase. Hemos aprendido a trabajar más rápido, a ponernos de acuerdo con nuestros compañeros y a exigirnos siempre un poco más".

Estudiante 7: "Lo que más me ha gustado ha sido el trabajo en grupo, en clase y las correcciones de las exposiciones que hemos realizado. Después el dinamismo de las clases".

Estudiante 8: "Tener que trabajar en grupo, recibiendo todos la misma nota, así todos nos esforzamos más. Poder aprender de nuestro errores a la hora de las presentaciones".

Los estudiantes también manifestaron su opinión sobre aspectos que podían ser mejorados. A continuación se reflejan, al igual que en el caso de las opiniones favorables, un resumen que recoge el sentir general.

Estudiante 9: "Me hubiera gustado recibir más comentarios que nos ayuden a mejorar a cada uno a nivel individual o incluso también nos ayuden en el trabajo en grupo". 
Estudiante 10: "El poco tiempo que teníamos para entregar ciertos trabajos. Esto, a veces hacía, que el resultado de dichos trabajos, no llegará a ser el mejor posible".

Estudiante 11: "Que la exposición de algunos trabajos que se realizaron en prácticas se hiciese el mismo día, pero entiendo que también es algo positivo porque nos ayuda a desarrollar otras capacidades".

Estudiante 12: "Tener que hacer un PowerPoint en 20 minutos (aunque luego muchas veces teníamos algo más de tiempo extra, así que no ha estado tan mal)".

Estudiante 13: "El poco tiempo para preparar las exposiciones".

Estudiante 14: "El agobio en algunas sesiones prácticas".

Estudiante 15: "Tener que hacer las cosas con muy poco tiempo. Hasta ahora nunca había preparado una exposición en 20 minutos".

Destacar que sólo uno de los estudiantes, manifestó como una de las cosas que menos le habían gustado de la asignatura (Estudiante 16) "Que las notas se compartan entre todos los integrantes del grupo".

Por otra parte, la Facultad de Ciencias Experimentales, entre sus planes de mejora contempla una actividad que consiste en solicitar un informe a los alumnos, que se canaliza a través de los delegados y subdelegados de los cursos, evaluando las diferentes asignaturas. A continuación se transcriben algunos de los párrafos del informe entregado por los estudiantes que han participado en esta experiencia docente.

Clases teóricas: Clases dinámicas y bien explicadas, haciendo que fueran interesantes... Clases prácticas: Excepcionalmente buenas. Aplicadas a la vida real: se aprendió a trabajar bajo presión, en equipo y a exponer en público... Finalmente, decir que esta asignatura estaba realmente adaptada al llamado Plan Bolonia, valorándose el trabajo diario del alumno...

\section{CONCLUSIONES Y DISCUSIÓN}

Todo docente con algo de experiencia sabe que no todas las promociones de estudiantes son iguales. Hay promociones realmente buenas, que se recuerdan con especial cariño y otras que no lo son tanto. También sabe que aplicando la misma metodología docente, hay grupos que funcionan perfectamente y otros en los que es necesario modificar sobre la marcha algunos aspectos, porque los estudiantes no colaboran, no aceptan la metodología originalmente elegida.

Cabe destacar que el grupo en el que se ha aplicado como metodología docente el trabajo colaborativo, además de ser reducido y haber elegido ellos la asignatura, ya que es optativa, se puede calificar como un grupo estupendo.

Pasada la primera sorpresa tras conocer la metodología que se iba a utilizar, que es totalmente distinta a la que están acostumbrados en el grado y el posible rechazo 
inicial al cambio, el grupo ha acogido con entusiasmo la metodología. Han participado en todas las actividades propuestas, de manera proactiva, incluidas las voluntarias (que no influían en la nota final).

Al final del cuatrimestre se han creado lazos de colaboración y amistad que no existían previamente, siendo un grupo cohesionado, dispuesto a ayudarse fuera del aula.

A pesar de la enorme carga de trabajo que ha representado para el docente (y también para los discentes) la experiencia, además de ser altamente enriquecedora en el aspecto personal, ha contribuido claramente al desarrollo personal de los estudiantes, resultando también muy positiva en el aspecto puramente cognitivo de alcanzar los objetivos de aprendizaje y las competencias planificadas.

\section{REFERENCIAS}

\section{Libros:}

Cabero, J. (2003). Principios pedagógicos, psicológicos y sociológicos del trabajo colaborativo: su proyección en la tele enseñanza. Redes de comunicación en la enseñanza. Barcelona: Paidós Ibérica S.A. Ediciones.

Serrano, J. M., y González-Herrero M. E. (1996). Cooperar para aprender. ¿Cómo implementar el aprendizaje cooperativo en el aula? Murcia: Diego Marín.

Slavin, R. E. (1995). Cooperative Learning: theory, research and practice (2nd Ed.) Boston: Allyn and Bacon.

\section{Recursos electrónicos:}

Echazarreta C., Prados F., Poch J. (2009). La competencia "el trabajo colaborativo": una oportunidad para incorporar las TIC en la didáctica universitaria. Descripción de una experiencia con la plataforma ACME (U de G), en Trabajo colaborativo, visiones disciplinarias. UOC Papers (8). Recuperado de http://www.uoc.edu/uocpapers/8/dt/esp/echazarreta prados poch soler.pdf

Heredia-Avalos, S., Moreno-Marín, J. C., Denton, C. D. y Calzado-Estepa, E. M. (2013). Trabajo en grupo en la docencia universitaria de titulaciones científicotécnicas, en XI Jornadas de Redes de Investigación en Docencia Universitaria. Retos de futuro en la enseñanza superior: docencia e investigación para alcanzar la excelencia académica Universidad de Alicante, Julio, 2013. Recuperado de http://web.ua.es/es/ice/jornadas-redes/documentos/2013-posters/335080.pdf

Ibarra-Sáiz M. S., Rodríguez-Gómez G. (2007). El trabajo colaborativo en las aulas universitarias: reflexiones desde la autoevaluación, en Revista de Educación (344), Páginas 355 - 375. Recuperado de http://www.revistaeducacion.mec.es/re344/re344 15.pdf 
Panitz, T. (1997). Collaborative versus Cooperative Learning: Comparing the Two Definitions Helps Understand the Nature on Interactive Learning, Cooperative Learning and College Teaching, (8). Recuperado de http://files.eric.ed.gov/fulltext/ED448443.pdf

Salinas J. (2004), Innovación docente y uso de las TIC en la enseñanza universitaria, en Revista de Universidad y Sociedad del Conocimiento (1). Recuperado de http://www.uoc.edu/rusc/dt/esp/salinas1104.pdf

Zubimendi-Herranz J. L., Ruiz-Ojeda M. P., Carrascal Lecumberri E., de la Presa Donado H. (2010). El Aprendizaje cooperativo en el aula universitaria: manual de ayuda al profesorado, en Publicaciones de la Universidad del País Vasco. Recuperado de http://www.industria-ingeniaritza-tekniko-bilbao.ehu.es/p229content/es/contenidos/noticia/euiti aprendizaje/es noticia/adjuntos/aprendizaje cooperativo aula universitaria.pdf 